\title{
Aerodynamic Model Analysis and Flight Simulation Research of UAV Based on Simulink
}

\author{
Chao Yun, Xiao-Min Li
}

Department of UAV Engineering, Ordnance Engineering College, Shijiazhuang, China.

Email: oec_ljw2009@163.com

Received November $24^{\text {th }}, 2012$; revised December $25^{\text {th }}, 2012$; accepted January $3^{\text {rd }}, 2013$

\begin{abstract}
Mathematical simulation method can be adopted to check flight characteristic of UAV, also can be adopted to simulate hardware experiments of unmanned aerial vehicle (UAV), then related flight experiments can be performed. The simulation method can reduce the flight periods, cost and risk. UAV flight model research play an important role in simulation and modeling in initialize periods of the UAV producing. The study of the paper focuses on the aerodynamic force modeling work of UAV based on Simulink. The designed model not only can afford mathematics simulation experiment but also will do benefits to the research of flight control, navigation guidance of UAV.
\end{abstract}

Keywords: Mathematical Simulation; Unmanned Aerial Vehicle (UAV); Aerodynamic Force; Simulink

\section{Introduction}

The UAV is getting more and more important with its increasing demand in war, now each country pays more attention to researching work of UAV, includes the early researching project, medium-term hardware simulation, performance validation, and simulation training. All above mentioned need the simulation research.

UAV plays more and more important role in modern war, which is a new form of the modern war. The researching work of kinematics characteristic is the basic work of the UAV system. The research work includes the simulation of flight track, guideline about and related techniques and tactics, the validation work through the simulation operation. Through high fidelity training for the UAV operator, UAV system will play more and more important role in its application field and heighten its fighting efficiency.

\section{Simulation Technology of System}

Simulation technology is based on conform principle, information technology and the technique of its application fields, it is a new all-around technology which is adopting computer and other physical equipments, and make use of related model to do the experiments [1]. System simulation is an experimental method to acquire information through using the system model or assumed system. The basic actions of system simulation are modeling, simulation modeling and simulation experiments and experimental analysis. It not only can offer us advanced methods to do the research working of analysis, decision-making, designing and training, but also increase the cognitive ability for the extension inherent rule. It will promote the progress of the subject form qualitative analysis to ration. Now System simulation technology is widely used in the following research areas [2].

\subsection{Application of System Analysis and Design}

The system simulation technology could do the argumentation and feasibility analysis for the unfounded system, and do the basic work for system design. In the course of the system designing it can do help in building the model, and optimization designing work; when the system is built, we could look for optimal control law for the system through analysis results of the system.

\subsection{Application of Theory Research}

The theory research of the system mainly depends on theoretic deducing in the past. Simulation technology can offer us the useful tool in the theory research, the tool not only can verify the validity of theory, but also can discover the system conflicts between theory and reality.

\subsection{Application of Professional Training and Education}

It is an important characteristic of the system simulation adopted in training and education. Now, simulation training systems have been adopted in many complicated equipments (e.g., chemical equipment electricity station, electricity netting) and carrying systems (e.g., airplane, 
motorcar and shipping). They can afford important assistance in heightening efficiency, saving cost and safety training.

\section{Aerodynamic Characteristic Analysis}

The flight simulation system designed in paper adopts modularize method. The primary module includes telecontrol and remote sensing modules, aerodynamic model, power equipment modules (includes engine model and propeller model), task equipment module and scene module. The aerodynamic module id discussed in the paper.

In above modules, tasks of telecontrol and the remote sensing module involves decoding the telecontrol command and code information of remote sensing according to frame format. Decode the control instructions and export them to the equipment about power device (rudder equipment), code the aircraft pose information and information of task equipment and fill the remote sensing into the frame.

Aerodynamic module calculates the propeller's thrust and torque according to deflexion of the rudder, and then the calculation results are put into flight dynamic equation module shown as Figure 1. The flight dynamic module is to calculate kinematics equations, the results of the position and pose will be sent to telecontrol and remote sensing module. Stabilization module is to strengthen the stability of the system. Through contrasting the setting date and module calculation data, then the system can give the order to rudder to avoid errors.

The core of the flight simulation system is a flight dynamic model. The flight simulation dynamic model is consisted of two parts, one part is computing UAV dynamic characteristic, which can gain the aerodynamic force and moment; the other part is to obtain kinematics parameters of UAV through calculating 6 parameters equation [3].

In this module (UAV dynamic simulation model module), It should consider all forces and moments includes gravity, aerodynamic force, engine thrust and various moments, etc. In the reference frame of airplane, motion acceleration and angle acceleration should be calculated,

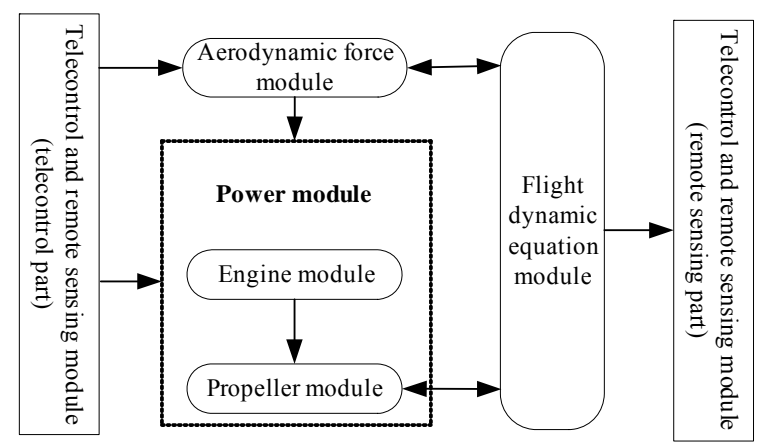

Figure 1. Aerodynamic module of UAV. we also can add wind disturb and turbulence into in aircraft reference frame, which are adopted to compute the parameters of flight track. The angle rate in an aircraft reference frame can be resolved and transformed into the ground reference frame, then attack angle and sideslip angle and their transformation ratio can be calculated, which would offer to aerodynamic module. The angle could be decompounded in ground reference frame and 3 Euler angles can be gained by integral, the computing results could offer attitude parameters to system. UAV 6 degrees of freedom (DOF) calculation flowchart is shown as Figure 2. In the paper, we adopt modularization method, aerodynamic module is one of the modules in the simulation system, when the structure of the airplane is changed, we can rebuild the aerodynamic force module, others module is unchanged.

\subsection{Aerodynamic Characteristic of UAV}

In the paper, study on a certain UAV which belongs to middle or miniature type. Its mass is less than 400 kilograms, the wingspan is about $7 \mathrm{~m}$, the wing area is $4 \mathrm{~m}^{2}$, flight height is less than $5000 \mathrm{~m}$, and the precise of the simulation system is according to different purpose. The complexity of mathematics model is different because the different simulation purpose. The flight height and speed is not higher in the project. So according to structure parameters and experimental purpose, using airplane dynamic simulation model in training simulator, we can make the assumption as below:

1) The airplane is rigid body, so we can neglect the elasticity effect on the body.

2) Mass and CG (center of mass) is constant.

3) Earth is inertia coordinate and assumed geography coordinate is inertial coordinate;

4) Neglect curvature of the earth.

5) Gravity is constant, as the UAV is flying altitude is not higher.

6) Assumed the $O X$ axis and $O Y$ axis is in the symmetry plane of coordinate, therefore $I_{X z}=I_{y z}=0$.

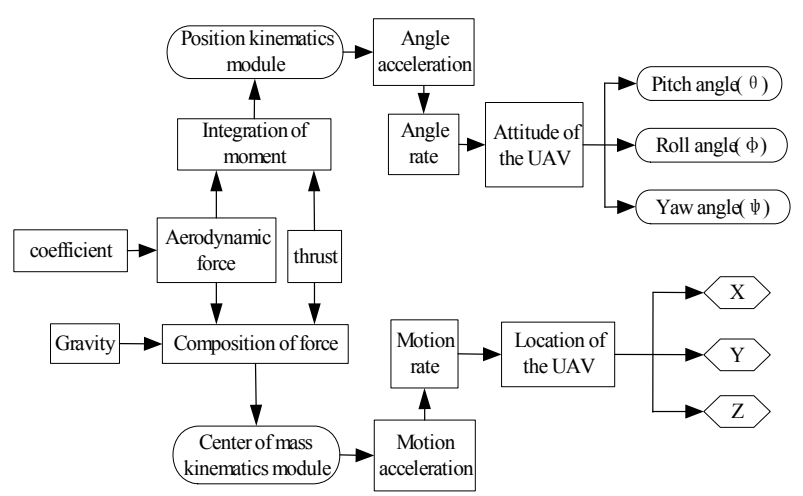

Figure 2. Calculation process of the kinematics equation with 6 parameters. 


\subsection{Characteristic Analysis of UAV}

\subsubsection{Dynamic Equation}

The forces of UAV in the air include gravity, aerodynamic force and thrust force by the engine. It is shown that established vector equation in the reference of aircraft is the simple and convenient Aircraft coordinate is a dynamic coordinate, it have displacement moment and rotating moment, the moment equation in aircraft coordinate is expressed as follows.

$$
\frac{\mathrm{d} V}{\mathrm{~d} t}=\frac{\delta V}{\delta t}+\Omega \times V
$$

$\frac{\mathrm{d} V}{\mathrm{~d} t}$ represents absolute derivative of vector $V$ in ground coordinate. $\frac{\delta V}{\delta t}$ represents relative derivative of vector $V$ in aircraft coordinates.

Its matrix expression is as follows:

$$
\left[\begin{array}{c}
V_{x} \\
V_{y} \\
V_{z}
\end{array}\right]=\left[\begin{array}{c}
\omega_{y} V_{z}-\omega_{z} V_{y} \\
\omega_{z} V_{x}-\omega_{x} V_{z} \\
\omega_{x} V_{y}-\omega_{y} V_{x}
\end{array}\right]+C_{a}^{t}\left[\begin{array}{c}
-X \\
Y \\
Z
\end{array}\right]+C_{g}^{t}\left[\begin{array}{l}
0 \\
0 \\
g
\end{array}\right]
$$

$C_{a}^{t}$ represents transfer matrix from velocity coordinate to aircraft coordinate.

$C_{g}^{t}$ represents matrix from ground coordinate to aircraft coordinate.

$X, Y, Z$ are aerodynamic forces of drag, lift and side force in rate reference frame.

The UAV can receive the moment in the air mostly includes aerodynamic moment, control moment, crossed moment, damp, etc.

It is shown that aircraft rotary equation, which surround the center of mass is most simply and convenient for analysis. It is assumed that aircraft coordinate is dynamic coordinate, its rotating angular rate is $\omega$, it can be calculated through (3).

$$
\frac{\mathrm{d} H}{\mathrm{~d} t}=\frac{\delta H}{\delta t}+\omega \times H
$$

Its matrix expression is shown as (4),

$$
\left[\begin{array}{c}
p \\
q \\
r
\end{array}\right]=I^{-1}\left(M_{s t}+M_{c}+M_{d}+M_{j}\right)
$$

In Equation (4), $I$ is rotating inertia moment, $M_{\mathrm{st}} M_{c}$ $M_{d} M_{j}$ are aerodynamic moment, control moment, damp moment and crossed moment. Projection of $M_{c}, M_{d}, M_{j}$ in aircraft coordinate together with $M_{s t}$ are consisted of multiple moment.

\subsubsection{Kinematics Equation}

The kinematics equation for the centroid of UAV is shown as (5),

$$
\left[\begin{array}{c}
V_{X} \\
V_{Y} \\
V_{Z}
\end{array}\right]=C_{a}^{g}\left[\begin{array}{c}
v_{x} \\
v_{y} \\
v_{z}
\end{array}\right]
$$

$C_{a}^{g}$ represents transfer matrix from rate coordinate to ground coordinates

Rotating equation of UAV moved around centroid, angular rate of rotating of UAV is established which is relative to ground coordinate [4].

$$
\left(\begin{array}{c}
\mathrm{d} \phi / \mathrm{d} t \\
\mathrm{~d} \theta / \mathrm{d} t \\
\mathrm{~d} \psi / \mathrm{d} t
\end{array}\right)=\left(\begin{array}{c}
\omega_{x b}+\tan \theta\left(\omega_{y b} \sin \phi+\omega_{z b} \cos \phi\right) \\
\omega_{y b} \cos \phi+\omega_{z b} \sin \phi \\
\left(\omega_{y b} \sin \phi+\omega_{z b} \cos \phi\right) / \cos \theta
\end{array}\right)
$$

$\omega_{x b}, \omega_{y b}, \omega_{z b}$ represent component of the flight angular rate in aircraft coordinate, respectively, the angular rate is determined by attitude angle's rate $\mathrm{d} \psi / \mathrm{d} t$, $\mathrm{d} \theta / \mathrm{d} t, \quad \mathrm{~d} \phi / \mathrm{d} t, \mathrm{~d} \psi / \mathrm{d} t$ is along $z_{g}$ axis, $\mathrm{d} \theta / \mathrm{d} t$ is along $y$ axis and $\mathrm{d} \phi / \mathrm{d} t$ is along $x$ axis.

Through related analysis we can simply the 6 degrees of freedom mathematics model for UAV, in order to keep the precise result we should keep the airspeed $V$ and attack angle $\alpha$ of UAV in linear region, which will keep facticity and reliability of the simulation.

\subsection{Dynamic Modeling Based on Simulink}

Simulink is a powerful toolbox for dynamic modeling. It has many advantages such as strong computing ability, the simpleness of the program, abundant repeating model, which can transform code in real time with high efficiency. It has an abundant toolbox about engineering, includes aerospace tool which is adopted in aircraft modeling and simulation. Simulink adopts module design method, which is very convenient and intuitionist to construct a dynamic model, Aerosim is a whole 6 degree of freedom modeling toolbox which is developed from the Unmanned Dynamics company in the Simulink environment [5-7]. Using these modules we can construct the simulation system, choose specific module which we need and then combine these modules according to dynamic data flow. Many modules cannot be used directly, for example, atmosphere model module, and physical geography model module and so on. UAV system inside structure is shown as Figure 3.

\section{Simulation Analysis}

Simulink and Aerosim toolbox offer several integrated airplane models, through configuring and setting aircraft's parameters, users can specify a certain type structure aircraft according to template aircraft configuration scripts, e.g., modify the parameter in Config_template.m 


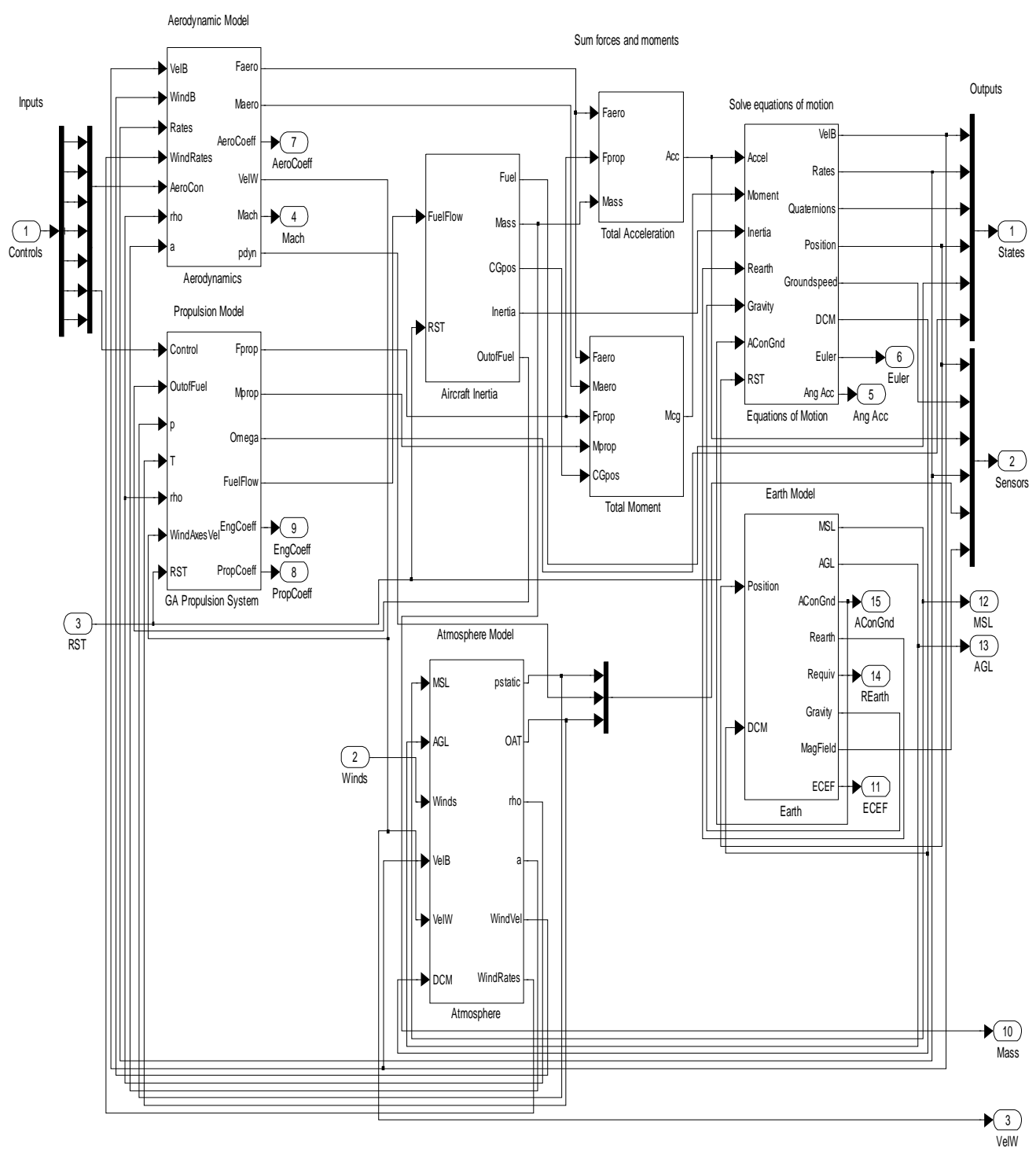

Figure 3. Module structure of the UAV system.

(includes aircraft dynamic, propulsion, wing span, inertial parameters, etc.). Running through this script at the Matlab command prompt, a new aircraft parameter file of the form filename.mat will be created [7-10]. If we choose this file with. mat extension and fill it into the UAV parameters setting dialog box, we will accomplish parameters configuration.

\subsection{Simulation Example One}

We make the assumption that the initial height of the UAV is $1000 \mathrm{~m}$, initial speed is [ 5000 , rudder offset is 0 and the aircraft in ideal conditions (i.e., it flies under no wind condition), add stabilization control loop Proportional-Integral-Differential (PID) control loop, we can see from simulation results that the airspeed is steady at $48 \mathrm{~m} / \mathrm{s}$ with the setting value, in other word, it flies steady with $48 \mathrm{~m} / \mathrm{s}$. pitch angle (theta) is steady at $17^{\circ}$ with setting value just shown as Figure 4.

\subsection{Simulation Example Two}

We make the assumption that flight initial conditions of example 2 are nearly the same as example 1 and only add wind vector $[5-50]$, i.e., add the lateral direction wind in the simulation. As known that UAV could encounter much complicated weather conditions in its flying, and the variation of air current will affect flight attitudes of UAV. So the research under wind environment has its practical meaning. In the simulation results in the Figure 5 , it can be found that each flight parameter is instability. The oscillate frequency of each parameter is accelerated, in control loop (PID control loop) the output will be stable at the setting value, but it will oscillate around the setting value, the condition is as same as the real environment. 

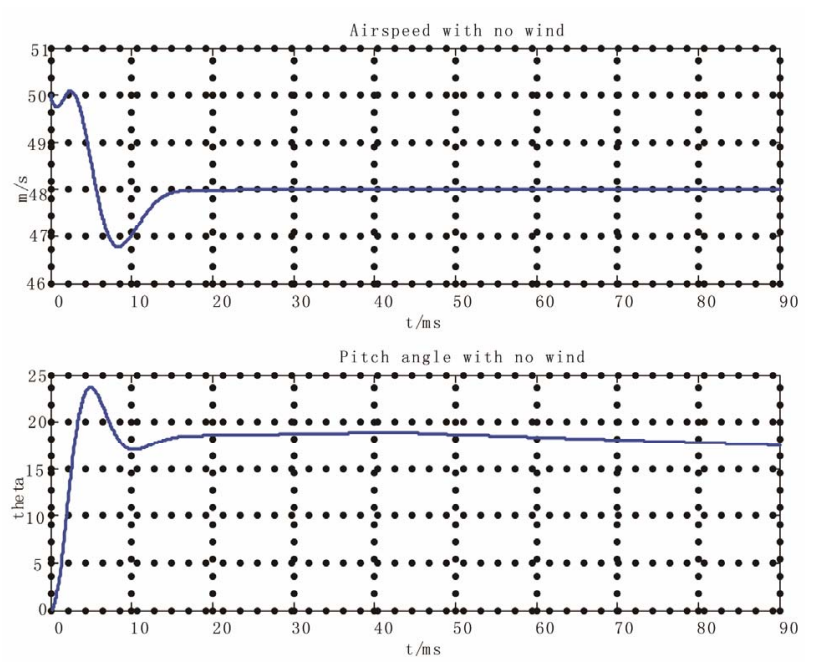

Figure 4. Output results of airspeed and angle of pitch.

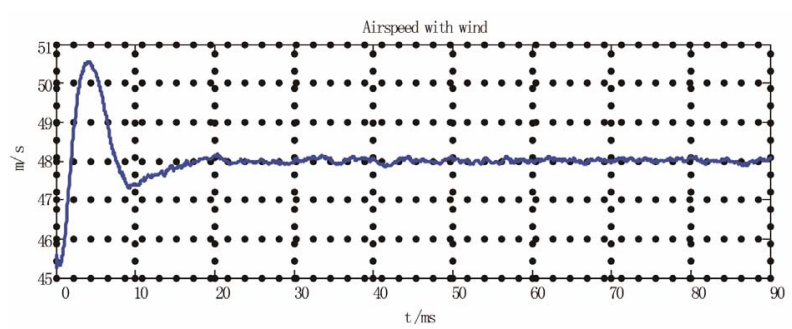

Pitch angle with wind

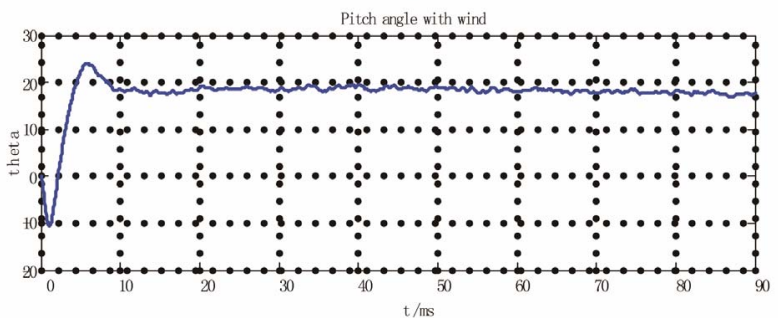

Figure 5. Output results of airspeed and angle of pitch.

\section{Conclusion}

In the paper we introduce the development tend and application fields of the simulation technology. We do the research work of aeronautic simulation of UAV flight control based on characteristic and study on 6 degrees of freedom aerodynamic model. Modeling and simulation according to the certain type based on Simulink/Aerosim toolbox are performed; simulation is done under ideal condition and wind environment. We also present simulation curve of airspeed and pitch angle. The related results show that the condition with wind is closer to actual condition, so our research work can offer the foundation for establishing UAV training simulator.

\section{REFERENCES}

[1] P. G. Fahlstrom and T. J. Gleason, "UAV System Introduction," 2003.

[2] B. L. Stevens and F. L. Lewis, "Aircraft Control and Simulation," 2nd Edition, Wiley, New York, 1992.

[3] K. Nonmi, "Prospect and Recent Research \& Development for Civil Use of Autonomous Unmanned Aircraft as UAV and MAV," Journal of system Design and Dynamics, Vol. 1, No. 2, 2007, pp. 120-128.

[4] N. Dai and Y.-L. Si, "Helicopter Flight Control System Modeling and Simulation by MATLAB," Journal of System Simulation, Vol. 18, No. 1, 2006, pp. 240-242.

[5] "Information on AeroSim Block User's Guide." http//: www.u-dynamics.com

[6] "Using SIMULINK," Version 6.5, The MathWorks, Inc., Natic.

[7] B. Xu and Z. Liu, "Matlab Use in Engineering and Mathematics," Tsinghua University Press, Beijing, 2000.

[8] L.-N. Wu and Y.-M. Huang, "Multi-Mode State Flow," Journal of Hangzhou Dianzi University, Vol. 25, No. 4, 2005, pp. 34-37.

[9] B. Stevens and F. Lewis, "Aircraft Control and Simulation,” John Wiley \& Sons, Inc., Hoboken, 1992.

[10] M.-L. Zhang, "Flight Control System," Aviation Industry Press, Beijing, 1983. 